\title{
The preventative effect of Akt knockout on liver cancer through modulating NF-kB-regulated inflammation and Bad-related apoptosis signaling pathway
}

\author{
BIN HU $^{1 *}$, MING SUN ${ }^{2 *}$, JIAJUN LIU ${ }^{3}$, GUOLIN HONG ${ }^{1}$ and QIN LIN ${ }^{4}$ \\ Departments of ${ }^{1}$ Laboratory Medicine, ${ }^{2}$ Reproductive Medicine, ${ }^{3}$ Infectious Diseases and ${ }^{4}$ Radiation Oncology, \\ Reproductive Medicine, The First Affiliated Hospital of Xiamen University, Xiamen, Fujian 361003, P.R. China
}

Received November 10, 2015; Accepted December 17, 2015

DOI: $10.3892 /$ ijo.2016.3383

\begin{abstract}
Primary liver cancer is globally the sixth most frequent cancer, and the second leading cause of cancer death and its incidence is increasing in many countries, thus, becoming serious threat to human health. Substantial research has focused on the treatment and prevention of liver cancer. However, the underlying molecular mechanism of liver cancer are still not fully understood, and therefore development of treatments are delayed. Akt has been suggested to play an essential role in the progression of inflammation response and apoptosis. Hence, in the present study, Akt knockout mice and cell lines were used as a model to investigate the molecular mechanism of Akt-associated inflammatory and apoptotic signaling pathway with $\mathrm{NF}-\kappa \mathrm{B}$ and $\mathrm{Bad}$ in the progression of liver cancer. Western blotting, quantitative RT-PCR (qRT-PCR), immunohistochemistry, ELISA and flow cytometric analysis were used to determine the key signaling pathway in the development of liver cancer. The results indicated that, compared to the normal liver cells, the expression of Akt was significantly higher in liver cancer cell lines. In addition, Akt-knockout liver cancer cells showed lower Akt expression. We also, found that Akt-knockout cancer cell lines modulated inflammation response and apoptosis via inhibiting $\mathrm{NF}-\kappa \mathrm{B}$ expression and suppressing apoptotic activation. Our results indicated that
\end{abstract}

Correspondence to: Dr Guolin Hong, Department of Laboratory Medicine, The First Affiliated Hospital of Xiamen University, Xiamen, Fujian 361003, P.R. China

E-mail: xmhg19899@sina.com

Dr Qin Lin, Department of Radiation Oncology, Reproductive Medicine, The First Affiliated Hospital of Xiamen University, Xiamen, Fujian 361003, P.R. China

E-mail: linqin05@163.com

*Contributed equally

Key words: liver cancer, Akt knockout, NF-кB, Bad, inflammation, apoptosis the downstream signals, including cytokines regulated by $\mathrm{NF}-\kappa \mathrm{B}$ signaling pathway and caspase-3-activated apoptosis affected by Bad were downregulated for knockout of Akt. These findings demonstrated that Akt is related to $\mathrm{NF}-\kappa \mathrm{B}$ and Bad signaling pathway possibly playing a direct role in the progression of liver cancer. Thus, Akt might be an important and potential treatment choice for the clinical diagnosis and treatment in the future.

\section{Introduction}

Liver cancer, also known as hepatic cancer, originates in the liver. Higher rates of liver cancer occur where hepatitis B and $\mathrm{C}$ are common, including East Asia and Sub-Saharan Africa (1). Viral infection with either hepatitis $\mathrm{C}$ virus (HCV) or Hepatitis B virus (HBV) is the chief cause of liver cancer in the world, accounting for $80 \%$ of hepatocellular carcinoma (HCC), with $>600,000$ deaths each year. The viruses cause HCC with massive inflammation, fibrosis and eventual cirrhosis within the liver. The current treatment strategies of liver cancer include surgical resection, radiotherapy and chemotherapy (2). However, many liver cancer patients fail to respond to initial chemotherapy, or they acquire drug resistance during the treatment (3). Normal cells divide as many times as needed and stop (4). They attach to other cells and stay in place in tissues. Cells become cancerous when they lose their ability to stop dividing. Normal cells will commit cell suicide (apoptosis) when they are no longer needed. Until then, they are protected from cell suicide by several protein clusters and pathways (5-7). However, liver cancer with high mortality rate, still has no effective measures for treatment. Therefore, the development of novel therapies for liver cancer is urgently needed.

The Akt pathway is a signal transduction pathway that promotes survival and growth in response to extracellular signals (8). Activated Akt mediates downstream responses, including cell survival, growth, proliferation, cell migration and angiogenesis, by phosphorylating a range of intracellular proteins. Studies have found that AKT pathway plays an important or protective role in many tumors, such as lung, breast, intestine cancer and pancreatic carcinoma (9). Sometimes the genes along these protective pathways are 
mutated, rendering the cell incapable of committing suicide when it is no longer needed. This is one of the steps that causes cancer in combination with other mutations. Thus, the Akt pathway is stuck in the position and the cancer cell does not commit suicide $(10,11)$. Akt can phosphorylate and activate the I $\mathrm{B}$ B kinase IKK- $\alpha$, causing degradation of I $\mathrm{B}$ and nuclear translocation of $\mathrm{NF}-\kappa \mathrm{B}$ where it promotes expression

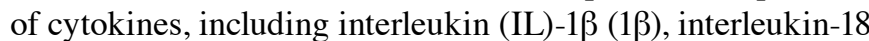
(IL-18), as well as tumor necrosis factor- $\alpha$ (TNF- $\alpha$ ), accelerating inflammation response. Akt, also, negatively regulates pro-apoptotic proteins by direct phosphorylation (12). For example, phosphorylation of Bad, the Bcl-2 family member, causes translocation from the mitochondrial membrane to the cytosol. Akt phosphorylates caspase-9, preventing a caspase cascade leading to cell death, modulating apoptosis. Thus, the association of Akt and NF- $\kappa \mathrm{B}$ and Bad signaling pathways play a critical role in the inflammatory formation and apoptotic development (13). We investigated the progression of liver cancer from $\mathrm{Akt} / \mathrm{NF}-\mathrm{\kappa B}$ and $\mathrm{Akt} / \mathrm{Bad}$ signaling pathway, revealing Akt-regulated inflammation and apoptosis during the development of hepatocellular carcinoma.

Hence, in the present study, in the first part we investigated whether Akt-knockout could affect liver cancer progression by interfering with $\mathrm{NF}-\kappa \mathrm{B}$ pathway activation, as well as Bad signaling pathway relating to inflammation and apoptosis, which might serve as a potential therapeutic target and prognostic marker for the treatment of liver cancer.

\section{Materials and methods}

Animals. Five-week-old BALB/c Akt-knockout mice and nude mice, were purchased from Vital River Laboratories (VRL; Beijing, China), and were used for a liver cancer xenograft model of MHCC97-H and Bel7402 cells. A total of $0.2 \mathrm{ml}$ medium containing 5 $10^{6} \mathrm{MHCC} 97-\mathrm{H}$ and Bel7402 cells was injected subcutaneously into the left and right posterior flank regions of each mouse. Mice were housed in a pathogen-free environment and tumor growth was examined each week (for a total of 5 weeks). Animals were euthanized and tumors were excised and subjected to pathological examination. The animal studies were approved by the First Affiliated Hospital Xiamen University, China.

Cell culture. Human liver cancer cell lines (MHCC97-H and Bel7402) and normal liver cells (hhl-5) were grown and maintained in RPMI-1640 medium containing 10\% fetal bovine serum (FBS) and 1\% penicillin/streptomycin. Recombinant lentiviral particles encoding Akt and short hairpin RNA targeting Akt were produced, concentrated and titrated. Cells were seeded at $3 \times 10^{4}$ cells $/ \mathrm{ml}$ and were infected with recombinant lentivirus twice with an interval of $12 \mathrm{~h}$ and incubated for $24 \mathrm{~h}$. After $24 \mathrm{~h}$, the medium was refreshed and the cells were cultured for another $24 \mathrm{~h}$.

ELISA measurement. At the end of the experiments, blood was extracted from the eyeball, the different serum concentration of the inflammatory cytokines TNF- $\alpha$, IFN- $\gamma$, IL-2, IL-1 $\beta$, IL-10, IL-18 and IL-6 were measured using ELISA kits according to the manufacturer's instructions (R\&D Systems, Inc., Minneapolis, MN, USA).
Inflammatory cell counts. The cell samples were centrifuged $\left(4^{\circ} \mathrm{C}, 3,000 \mathrm{rpm}\right.$ for $\left.10 \mathrm{~min}\right)$ to pellet the cells. The cell pellets were resuspended in PBS for the total cell counts using a hemacytometer, and cytospins were prepared for differential cell counts by staining with the Wright-Giemsa staining method.

Histopathological examination of cells and tissues. Histopathological evaluation was performed on mice and cells that were collected. Samples were fixed with $10 \%$ buffered formalin, imbedded in paraffin and sliced. After hematoxylin and eosin (H\&E) staining, pathological changes of tissues were observed under a light microscope. Some samples were also subjected to immunohistochemical staining (Akt, NF- $\kappa \mathrm{B}$ and Bad) according to CST Technology Co. introductions, and performed by Shanghai Zhenda Biotechnology, Co., Ltd. (Shanghai, China).

Immunofluorescence staining. After induction by conditioned culture medium, the cells were fixed in $4 \%$ paraformaldehyde, permeabilized with $0.1 \%$ Triton X-100 in PBS containing $0.5 \%$ BSA (PBS-BSA) for $30 \mathrm{~min}$. The cells were subsequently incubated with Akt, NF- $\mathrm{B}$ and Bad for $30 \mathrm{~min}$, followed by labeling with Alexa Fluor 488-conjugated rabbit anti-mouse or goat anti-rabbit IgG antibody. The cells were viewed under a fluorescent microscope.

Apoptosis analysis by terminal deoxynucleotidyl transferasemediated dUTP nick end labeling (TUNEL). Apoptosis assay of samples was determined by TUNEL used an In Situ Cell Death Detection Kit, Fluorescein (Roche Applied Science, South San Francisco, CA, USA) according to the manufacturer's protocol. The number of TUNEL-positive cells was counted under a fluorescence microscope. The percentages of apoptotic cells were calculated from the ratio of apoptotic cells to total cells. Tissue sections were counterstained with hematoxylin, then mounted and observed under light microscopy. The experiment was performed independently three times for each cell line.

Western blot analysis. Cell proteins were extracted using T-PER Tissue Protein Extraction reagent kit (Thermo Fisher Scientific) according to the manufacturer's instructions. Protein concentrations were determined by BCA protein assay kit, and equal amounts of protein were loaded per well on a $10 \%$ sodium dodecyl sulphatepolyacrylamide gel. Subsequently, proteins were transferred onto polyvinylidene difluoride membrane. The resulting membrane was blocked with Tris-buffered saline containing $0.05 \%$ Tween-20 (TBS-T), supplemented with 5\% skim milk (Sigma) at room temperature for $2 \mathrm{~h}$ on a rotary shaker followed by TBS-T washing. The specific primary antibody, diluted in TBST, was incubated with the membrane at $4^{\circ} \mathrm{C}$ overnight. Subsequently, the membrane was washed with TBS-T followed by incubation with the peroxidase-conjugated secondary antibody at room temperature for $1 \mathrm{~h}$. The immunoactive proteins were detected by using an enhanced chemiluminescence western blotting detection kit. The bands were observed using GE Healthcare ECL western blotting analysis system and exposed to X-ray film (Kodak). 
Table I. Primer sequences of RT-PCR analysis.

\begin{tabular}{lll}
\hline Gene & \multicolumn{1}{c}{ Forward primers (5'-3') } & \multicolumn{1}{c}{ Reverse primers (5'-3') } \\
\hline GAPDH & CATTCAAGACCGGACAGAGG & ACATACTCAGCACCAGCATCACC \\
Akt & GTGTCCAGTGTAGAATGACTC & ATCTGTCGGAGAACACACATG \\
NF-kB & GCAAAGGGAACATTCCGATAT & GCGACATCACATGGAAATCTA \\
IKK- $\alpha$ & GAACCGGCACCTGACACC & ACGACCTTCGTCAGTACCGA \\
IкB- $\alpha$ & AGCACAAAGAGAGTGTCGC & CGTCAGTCAGTGTGTATG \\
IL-1 $\beta$ & GACAGCAAAGTGATAGGCC & CGTCGGCAATGTATGTGTTGG \\
IL-18 & GCAGCAGGTGAGTGGGCAGT & CTGTACGCCTGGTTCGCTCTGT \\
Bcl-xL & CATGCTGGGGCCGTACAG & TTGTCCGACCTTTGGCAACT \\
Cytochrome $c$ & CAGAAGGAAGTTAGGCC & CGTCGCAGTGGATGATGTG \\
Apaf-1 & CTTCTCACTGTCGACTACCGC & GCGTCTCCTGTGCATTCG \\
Caspase- 9 & GACTCTTCCTGGTCTTACCATATT & CTGCTATTGCAAGGACCCAATT \\
Caspase-3 & GCAAGGACAAGATTCGATACT & GCCAGACTACATGGAAATCTA \\
\hline
\end{tabular}

Real-time quantitative PCR analysis. Total RNA isolated from an individual mouse kidney with TRIzol reagent (Invitrogen, Carlsbad, CA, USA) following the manufacturer's protocol, was evaluated for mRNA expression of

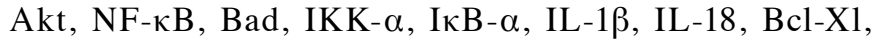
cytochrome $c$, Apaf-1, caspase-9, caspase-3 and glyceraldehyde 3-phosphate dehydrogenase (GAPDH). For reverse transcription, the SuperScript First-Strand Synthesis kit (RT-PCR; Invitrogen) was used. The primers for real-time PCR are summarized in Table I. All primer sequences were checked in GenBank to avoid inadvertent sequence homologies. They were designed and synthesized by BioGenes GmbH (Berlin, Germany). Reactions were performed using SYBR-Green PCR Master Mix (Applied Biosystems) in a Roche LightCycler 480 detection system. As an internal control, GAPDH levels were quantified in parallel with target genes. Normalization and fold change for each of the genes were calculated using the $2^{-\Delta \Delta \mathrm{CT}}$ method. The sequences of the genes are listed in Table I.

Secondary colony-forming assay. Briefly, cancer cells were suspended in $0.9 \%$ methylcellulose-based semisolid medium MethoCult H4100 (StemCell Technologies, Inc., Beijing, China). After 14 days, individual primary clones (450 cells) were trypsinized and re-plated in the same conditions to examine the secondary colonyforming ability for selfrenewal.

Flow cytometric analysis. For flow cytometric analysis, the hepatocytes were obtained through shearing liver tissue, separating by collagenase type II (Invitrogen) digestion and suspended in RPMI-1640 medium (Gibco-BRL). Cell suspensions were centrifuged at $1,000 \mathrm{rpm}$ for $5 \mathrm{~min}$ to remove cellular debris and impurities. Then, the hepatic mononuclear cells (MNCs) were harvested and re-suspended in $70 \%$ percoll (Sigma). MNCs were collected from the interphase, and washed twice in Hank's buffer (Gibco-BRL). According to the protocol of R\&D kit systems (R\&D Systems) for flow cytometry, anti-CD4 FITC and anti-CD8 FITC antibodies were added to the flow cytometry tube containing single-cell suspension, and the cells were analyzed by Cytomics $^{\mathrm{TM}} \mathrm{FC}$ 500 MCL of Beckman-Coulter (Beckman-Coulter, Inc., Brea, CA, USA).

Statistical analysis. Data are expressed as means \pm SEM. Treated cells, tissues and the corresponding controls were compared using GraphPad Prism (version 6.0; GraphPad Software, Inc., La Jolla, CA, USA) by a one-way ANOVA with Dunn's least significant difference tests. Differences between groups were considered significant at $\mathrm{P}<0.05$.

\section{Results}

Akt is overexpressed in liver cancer cell lines and tissues. In the present study, we evaluated the Akt expression in liver cancer cell lines and normal liver cells. As shown in Fig. 1A-C, we found Akt overexpressed in liver cancer cell lines compared to the normal cells via western blot analysis and RT-PCR analysis, demonstrating that Akt is likely to play an essential role in the progression of liver cancer. Thus, we aimed at Akt-knockout, and studied Akt expression in MHCC97-H (Fig. 1D and F) and Bel7402 cells (Fig. 1E and G). In order to confirm the role of Akt on liver cancer, immunohistochemical staining analysis was used to demonstrate the difference between the normal group, and tumor group, which showed higher survival percent of low Akt levels (Fig. 1H). Immunofluorescence staining was performed to further indicate Akt expression in NC group and $\mathrm{Akt}^{-/}$group of MHCC97-H and Bel7402 cells (Fig. 1I). These results suggested that Akt was expressed highly in liver cancer cell lines and after knockout of Akt, the survival percent of cells was improved compared to the normal cells, suggesting that Akt might be considered as a target for the treatment of liver cancer.

The effects of Akt on the progression of liver cancer. H\&E staining was applied to reveal the role of Akt-knockout in MHCC97-H and Bel7402 cells (Fig. 2A-C), which showed that 
A

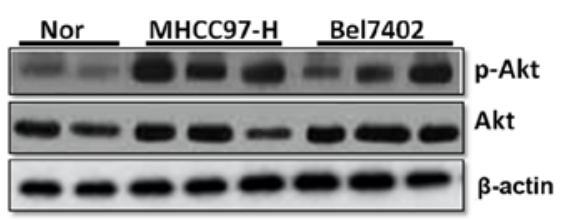

B

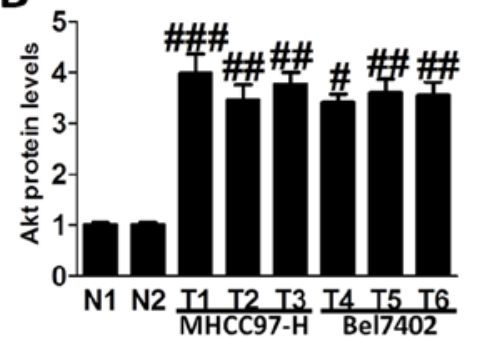

C

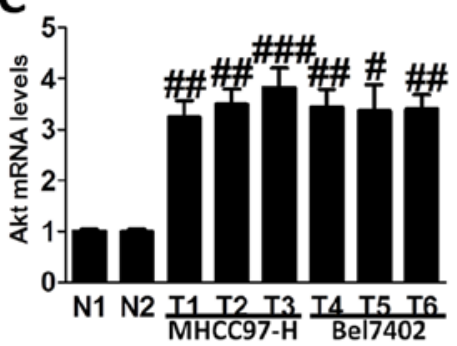

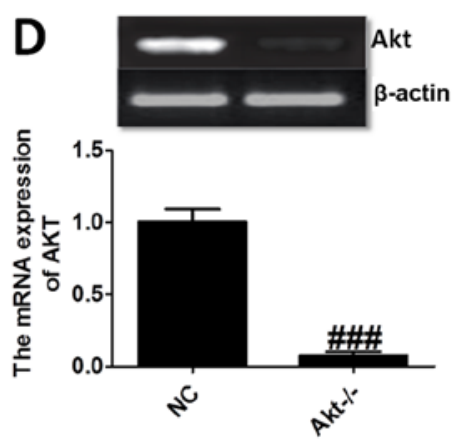
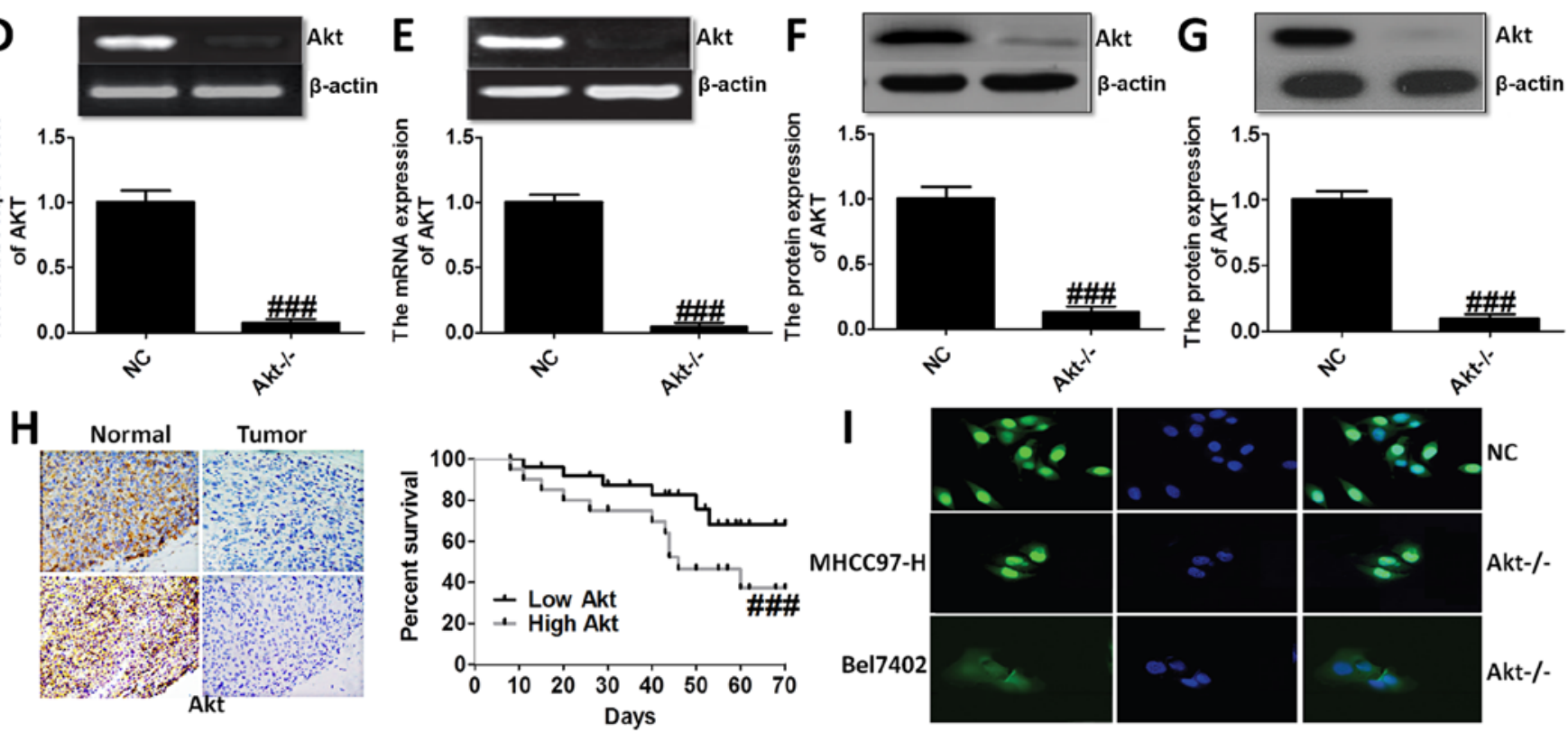

Figure 1. The expression of Akt in various cells. (A) Western blot analysis of Akt; (B) Akt protein levels in normal liver cells and liver cancer cell lines; (C) Akt mRNA levels in normal liver cells and liver cancer cell lines; (D) Akt mRNA levels after treatment of Akt knockout in MHCC97-H cells; (E) Akt mRNA levels after treatment of Akt knockout in Bel7402 cells; (F) Akt protein levels after treatment of Akt knockout in MHCC97-H cells; (G) Akt protein levels after treatment of Akt knockout in Bel7402 cells; (H) IHC staining of normal liver cells and tumor cells using Akt-specific antibodies; (I) IF analysis of NC group and liver cancer cell lines. Data are expressed as the mean $\pm \mathrm{SEM},{ }^{\#} \mathrm{P}<0.05,{ }^{\# \#} \mathrm{P}<0.01,{ }^{\# \# \#} \mathrm{P}<0.001$ vs. NC (control group).

Akt-knockout significantly decrease the number of invading cells. In addition, loss of Akt activity attenuated liver cancer cell growth in vitro, and the clone number of cells is shown in Fig. 2D and E. Moreover, nude mice were used to test the role Akt on cancer development directly, which indicated that compared to the NC group, mice treated with Akt-knockout of MHCC97-H and Bel7402 cells showed markedly lower tumor volume (Fig. 2F). After treatment, we tested the cell counts. The results showed that the number of cell counts in culture from 12 to $96 \mathrm{~h}$ was highly reduced in $\mathrm{Akt}^{-/-}$cells, in both MHCC97-H (Fig. 2G) and Bel7402 cells (Fig. 2H). The results proved that Akt is of great importance for the progression and development of liver cancer.

The effects of Akt on NF- $\kappa B$-regulated inflammation response. The knockout of Akt inhibited $\mathrm{NF}-\kappa \mathrm{B}$ expression was assessed using western blot and RT-PCR analysis with significant statistical difference compared to the control group (Fig. 3A and B). The immunofluorescence (Fig. 3C) and immunohistochemical staining (Fig. 3D) analysis showed that NF- $\mathrm{B}$ liver cancer cells were markedly decreased in both cell lines. Moreover, the transcriptional activity of $\mathrm{NF}-\kappa \mathrm{B}$ was determined by the luciferase assay when Akt was knocked out. Fig. $3 \mathrm{G}$ shows that $\mathrm{NF}-\kappa \mathrm{B}$ transcriptional activity was reduced markedly compared to the $\mathrm{NC}$ group. In addition, the upstream and downstream signals of NF- $\kappa \mathrm{B}$ were determined by western blot analysis, and RT-PCR demonstrating that cytokines, involving IL-1 $\beta$ and IL-18, were downregulated greatly, in accordance with the trend of $\mathrm{NF}-\kappa \mathrm{B}$ alteration (Fig. 3E and F).

Furthermore, we evaluated the levels of inflammatory cytokines in the serum of animal models. As shown in Fig. 4A, TNF- $\alpha$, IL-1, IL-6, IL-10, IL-1 $\beta$, IL-2, IL-18, IL-4, IL-17, IFN- $\gamma$, TGF-b1, TGF-b2 and VEGF were tested through ELISA kits. The results showed obvious cytokine reduction in Akt-knockout mice compared to the normal liver cancer mice. These results indicated that Akt regulated liver cancer progression via NF- $\kappa \mathrm{B}$ activation and its downstream signals contributed to the inflammation response.

We investigated TLR4/MyD88 signaling pathway contribution to the activation of NF- $\kappa \mathrm{B}$. The results showed significant reduction of TLR4 and MyD88 via western blot analysis and RT-PCR analysis in both MHCC97-H and Bel7402 cells (Fig. 4B). In addition, the downstream signals of TLR4/MyD88 pathway were decreased, including IRAK1, IRAK4 as well as TRAF6 (Fig. 4B and C), which was 
A

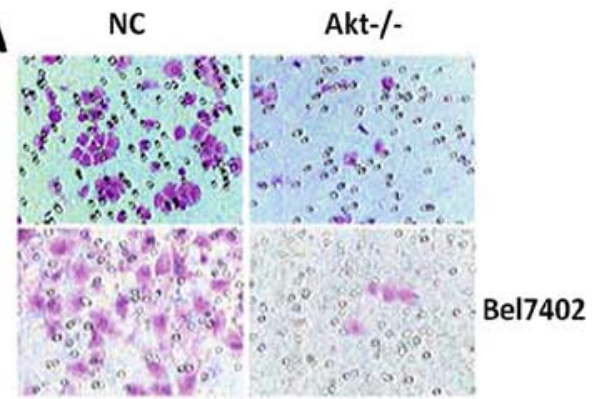

B

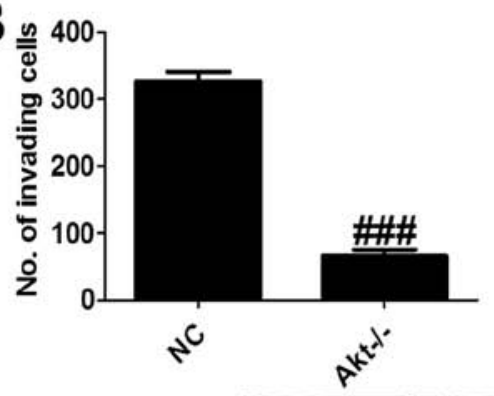

C

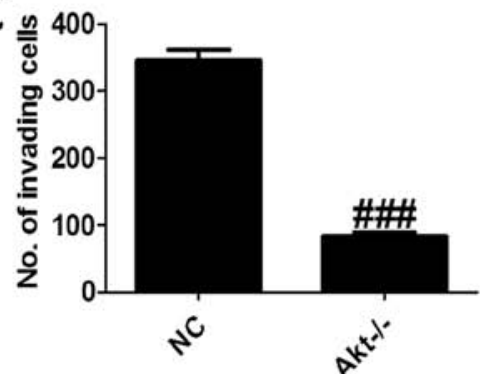

D
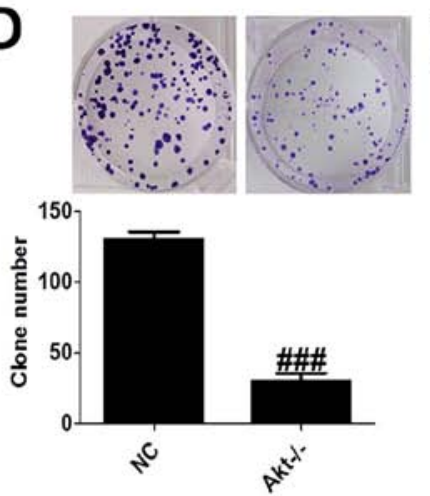

G

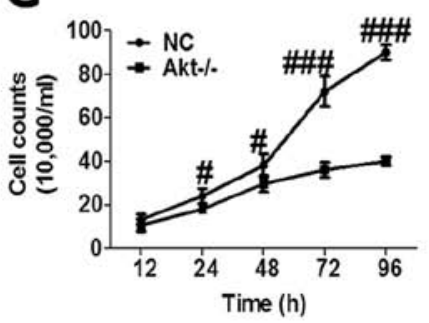

E
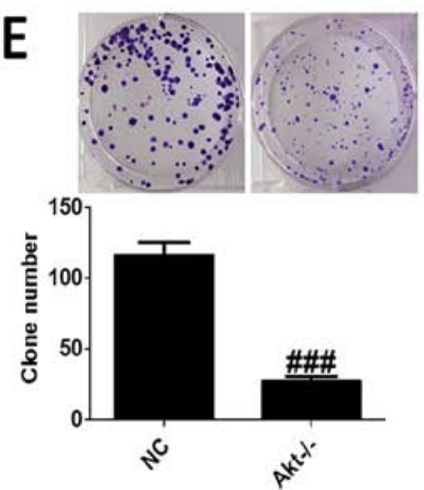

$\mathrm{H}$

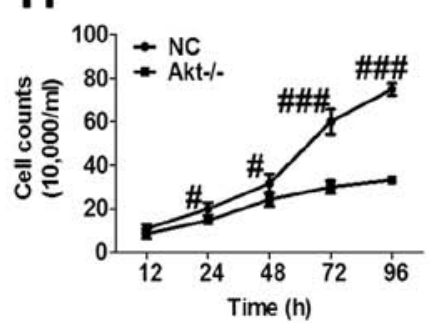

$\mathbf{F}$
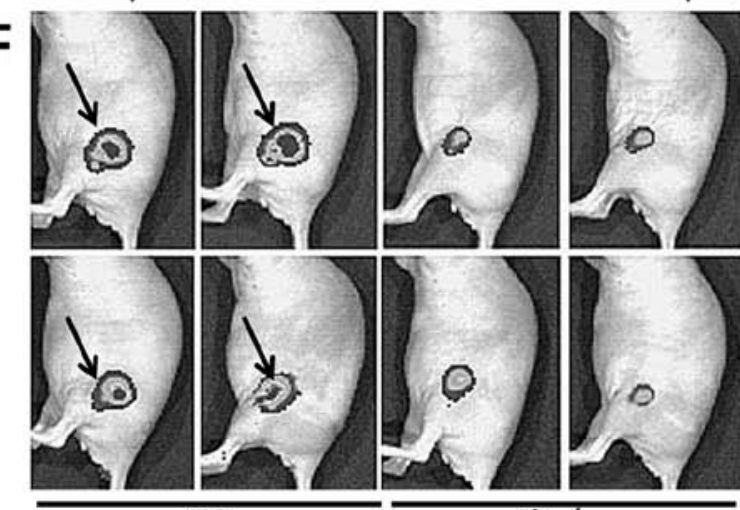

Bel7402

Figure 2. The effects of Akt on the progression of liver cancer. (A) IHC staining analysis of Akt ${ }^{-/}$in liver cancer cell lines; (B) the number of invading cells in MHCC97-H cells; (C) the number of invading cells in Bel7402 cells; (D) the clone number of MHCC97-H cells; (E) the clone number of Bel7402 cells; (F) tumor volume was measured using calipers; (G) cell counts after treatment of Akt-knockout in MHCC97-H cells; (H) cell counts after treatment of Aktknockout in Bel7402 cells. Data are expressed as the mean $\pm \mathrm{SEM},{ }^{\#} \mathrm{P}<0.05,{ }^{\# \#} \mathrm{P}<0.01,{ }^{\# \#} \mathrm{P}<0.001$ vs. NC group.

consistent with the expression trend of NF- $\mathrm{B}$ and inflammation-related cytokines. In this regard, we finally tested the expression level of PI3K in both cell types, displaying reduced phenomenon (Fig. 4B and C). These results possibly suggest to us that TLR4/MyD88 signaling pathway was regulated, in turn, after the overexpression of inflammation-associated factors. Activated PI3K phosphorylated Akt, leading to aggravation of inflammation response and apoptosis, which was likely to form a vicious cycle. Thus, knockout of Akt is related to the progression of the inflammation response.

The effects of Akt on Bad-regulated apoptosis in liver cancer. We supposed that apoptosis accelerates the progression of liver cancer. Thus, Bad, which is essential to apoptosis, was tested via immunofluorescence staining (Fig. 5A) and immunohistochemical staining analysis (Fig. 5B). The results showed that cells expressing Bad was higher in Akt-knockout cells. In addition, mice without Akt expression displayed serious cell death in liver cancer cells, as shown in Fig. 5C and D. Hierarchical clustering of microarray data from MHCC97-H and Bel7402 cells treated with Akt-knockout was analyzed. Additionally, Kaplan-Meier survival plot of liver cancer animals comparing a positive correlation with the Akt-knockout gene signature or negative correlation with the Akt-knockout gene signature was prepared, and is shown in Fig. 5E, demonstrating that the gene signature produced by Akt-knockout conferred a marked improvement in survival.

Furthermore, RT-PCR and western blot analysis were used to analyze Bad expression (Fig. 6A and B). The trend of Bad expression was consistent with immunofluorescence staining (Fig. 5A) and immunohistochemical staining (Fig. 5B), further indicating that Akt-knockout played a critical role in regulating Bad. On the contrary, we investigated the downstream signals of Bad, including Bcl-2, cytochrome $c$, Apaf-1, caspase-9 and caspase-3 (Fig. 6C and D). Akt-knockout induced significant reduction of the genes, except the upregulation of $\mathrm{Bcl}-2$, which plays an important role in anti-apoptosis in MHCC97-H and Bel7402 cells. Determined by Annexin V/PI double staining analysis, we found that knock out of Akt could suppress overexpression of Akt-induced apoptosis in liver cancer cells, showing higher apoptosis ratio in the indicated cancer cells (Fig. 6E). Moreover, caspase- 3 activation assay with the CaspGLOW fluorescein active caspase-3 staining kit with FACS analysis 


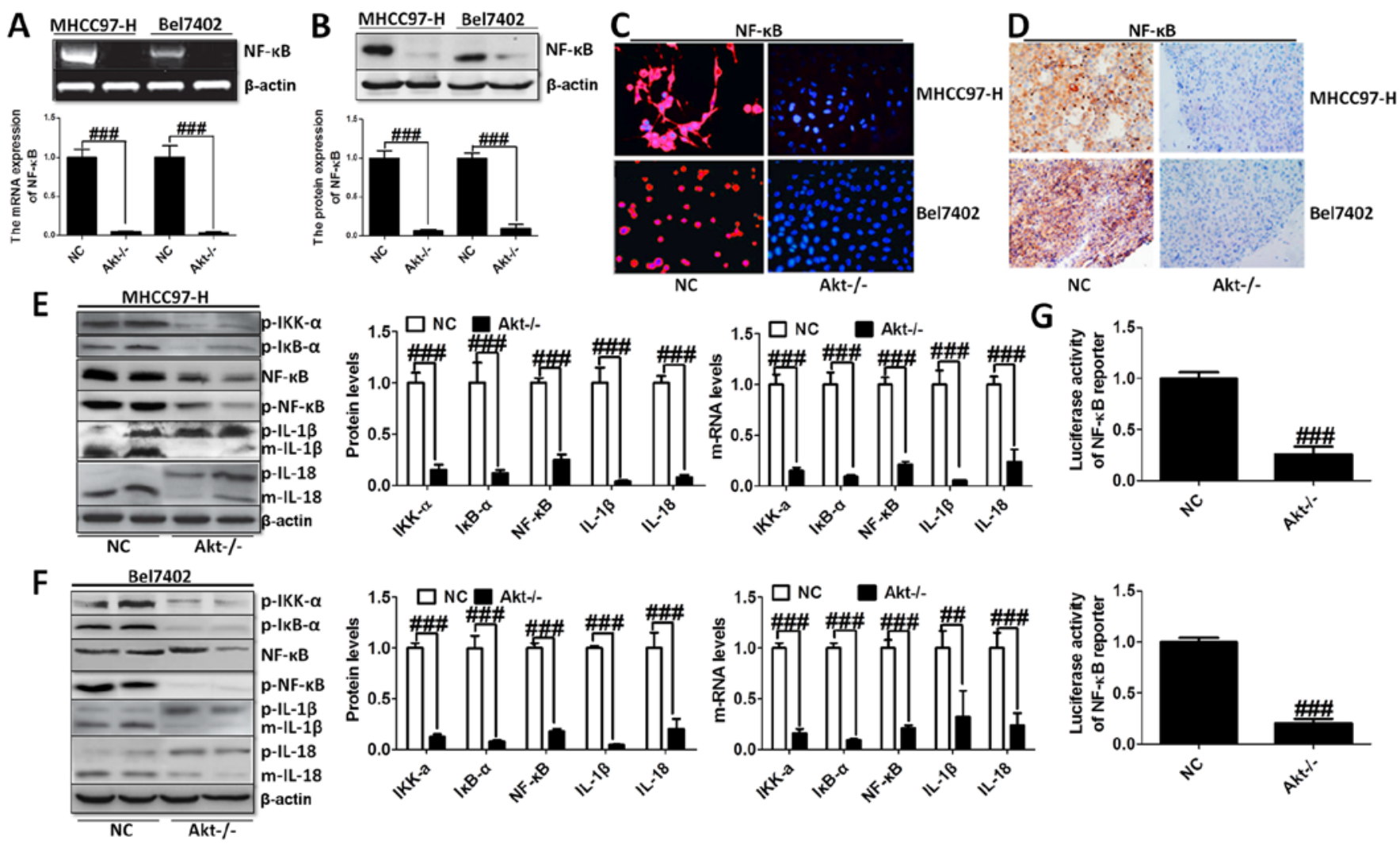

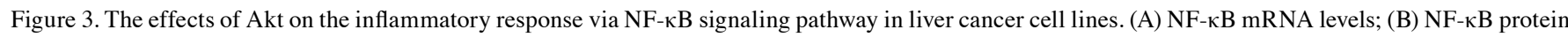

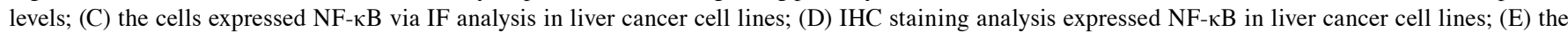
expression of NF- $\mathrm{kB}$ signaling pathway by western blot analysis and RT-PCR analysis in MHCC97-H cells; (F) the expression of NF- $\mathrm{kB}$ signaling pathway by western blot analysis and RT-PCR analysis in Bel7402 cells; (G) the transcriptional activity of NF- $\mathrm{BB}$ determined by the luciferase assay when Akt was knocked out in MHCC97-H and Bel7402 cells. Data are expressed as the mean $\pm \mathrm{SEM},{ }^{\# \#} \mathrm{P}<0.01,{ }^{\# \# \#} \mathrm{P}<0.001$ vs. NC group.

was applied to determine the effect of Akt on apoptosis development, showing that Akt-knockout cells had higher expression of caspase-3, which resulted in apoptosis in liver cancer cells (Fig. 6F). These data demonstrated that Akt-knockout serves as a survival signal and induction of apoptosis in cancer cells.

\section{Discussion}

Liver cancer is the third leading cause of cancer death after lung and stomach cancer world-wide. Liver cancer has been emphasized as a serious threat to human health $(14,15)$. Survival rates in the developed world are high, however, in developing countries survival rates are still poor, and unsatisfactory. Moreover, the formation and development of liver cancer is associated with high morbidity and mortality $(16,17)$. In previous research, some natural products have been used in cells, in animal experiments, and partly tested in clinical studies for preventing liver cancer providing useful information (18). However, the detailed underlying molecular mechanisms controlling liver cancer are still not fully explained. In addition, we are looking for the most effective treatment for liver cancer-related diseases.

Akt is known to be implicated in several types of cancer, including glioblastoma, ovarian, pancreatic and breast cancer. Studies have also found that Akt is upregulated in terms of mRNA production in the liver (19). The human liver cancer cell lines MHCC97-H and Bel7402, and the normal liver cells hhl-5 were used to investigate the expression of Akt in different cell lines (20). We found that the expression of Akt in liver cancer cell lines was higher compared to in the normal liver cells (Fig. 1A-C). Akt-knockout liver cancer cells displayed lower expression of Akt (Fig. 1D-I), which was used to further indicate the effect or importance of Akt in the treatment of liver cancer. Moreover, we found that the invading cells, clone number of liver cancer cells and the cell counts cultured for different times were downregulated after the knockout (Fig. 2A-H). Furthermore, xenografts in nude mice suggested that the volume of the tumor in Akt-knockout mice was ameliorated (Fig. 2F). The above results showed that Akt is of great importance in the development or progression for liver cancer. The activation of Akt further enhanced the NF- $\mathrm{B}$ pathway phosphorylation levels. As previously reported (21) the increase of NF- $\kappa \mathrm{B}$ pathway phosphorylation leads to inflammatory responses and cytokines production. Inflammatory abnormalities are a large group of disorders that underly a vast variety of human diseases, especially regulated by interleukins. Although the processes involved are identical to tissue inflammation, systemic inflammation is not confined to a particular tissue but involves the endothelium and other organ systems. The RT-PCR, western blot analysis, immunofluorescence, immunochemistry and luciferase activity analysis also provided evidence (Fig. 3). The results showed that the expression of 


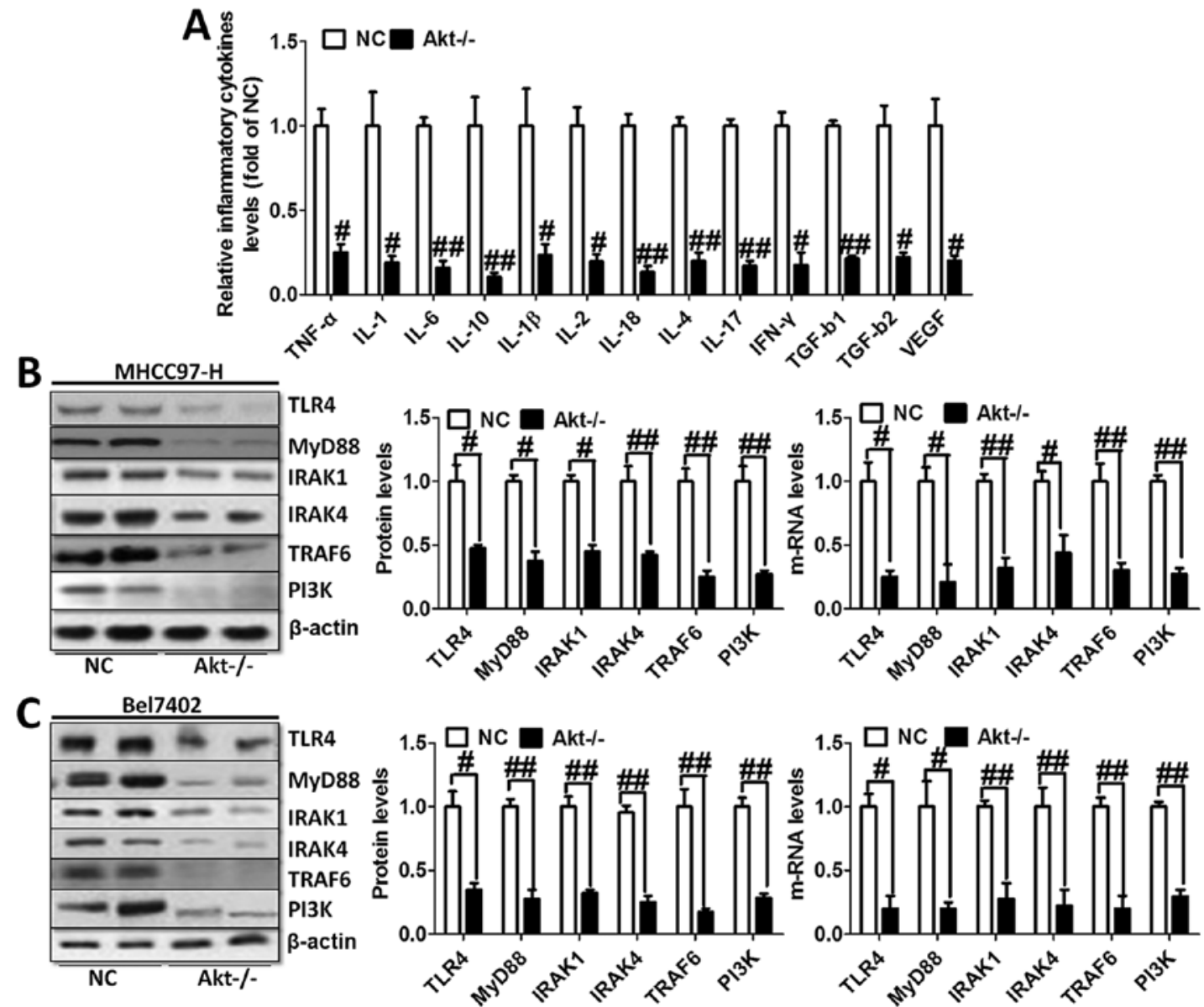

Figure 4. The effects of Akt on the inflammatory response via regulating cytokines and TLR4 signaling pathway. (A) Akt-knockout on the inflammatory response via regulating cytokines; (B) the effect of Akt-knockout on TLR4 signaling pathway via western blot analysis and RT-PCR analysis in MHCC97-H cells; (C) the effect of Akt-knockout on TLR4 signaling pathway via western blot analysis and RT-PCR analysis in Bel7402 cells. Data are expressed as the

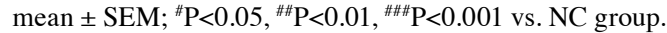
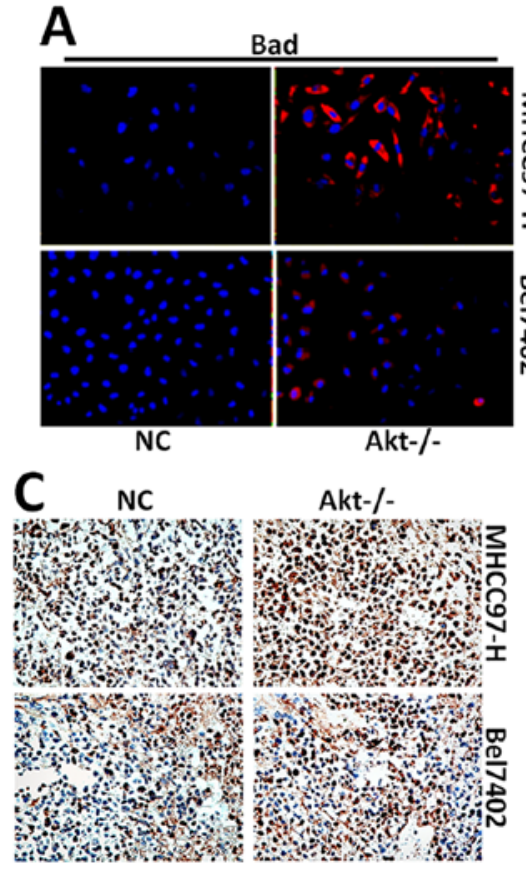

B

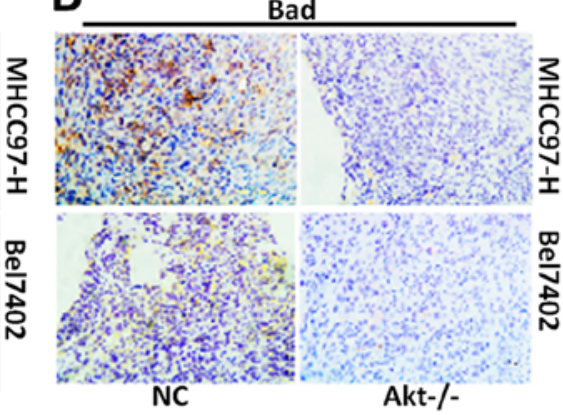

D NC

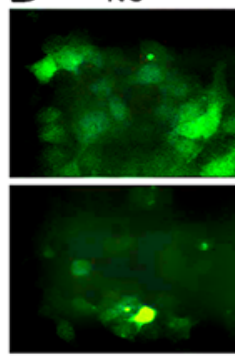

Akt-/-

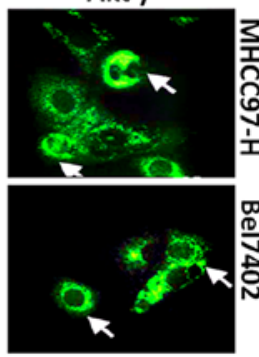

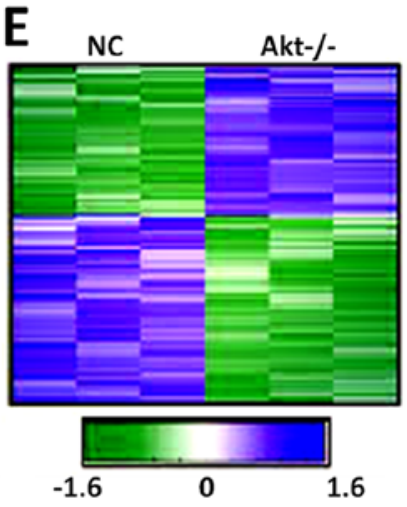

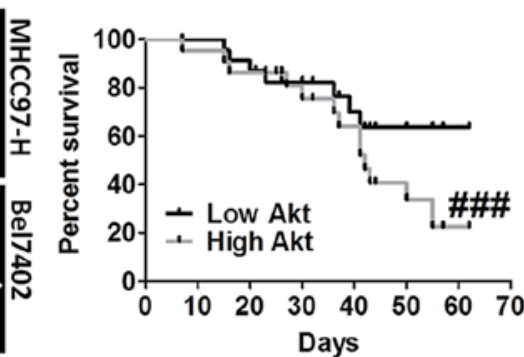

Figure 5. The effects of Akt on apoptosis via Bad signaling pathway in liver cancer cell lines. (A) The cells expressed Bad via IF analysis in liver cancer cell lines; (B) IHC staining analysis expressed Bad in liver cancer cell lines; (C) apoptotic cells determined by TUNEL assay in different treatment groups; (D) Akt-knockout induced punctuative distribution of Bad in liver cancer cell lines; (E) hierarchical clustering of microarray data from MHCC97-H and Bel7402 cells treated with Akt-knockout was analyzed. Data are expressed as the mean $\pm \mathrm{SEM} ;{ }^{\# \# ~} \mathrm{P}<0.001$ vs. NC group. 

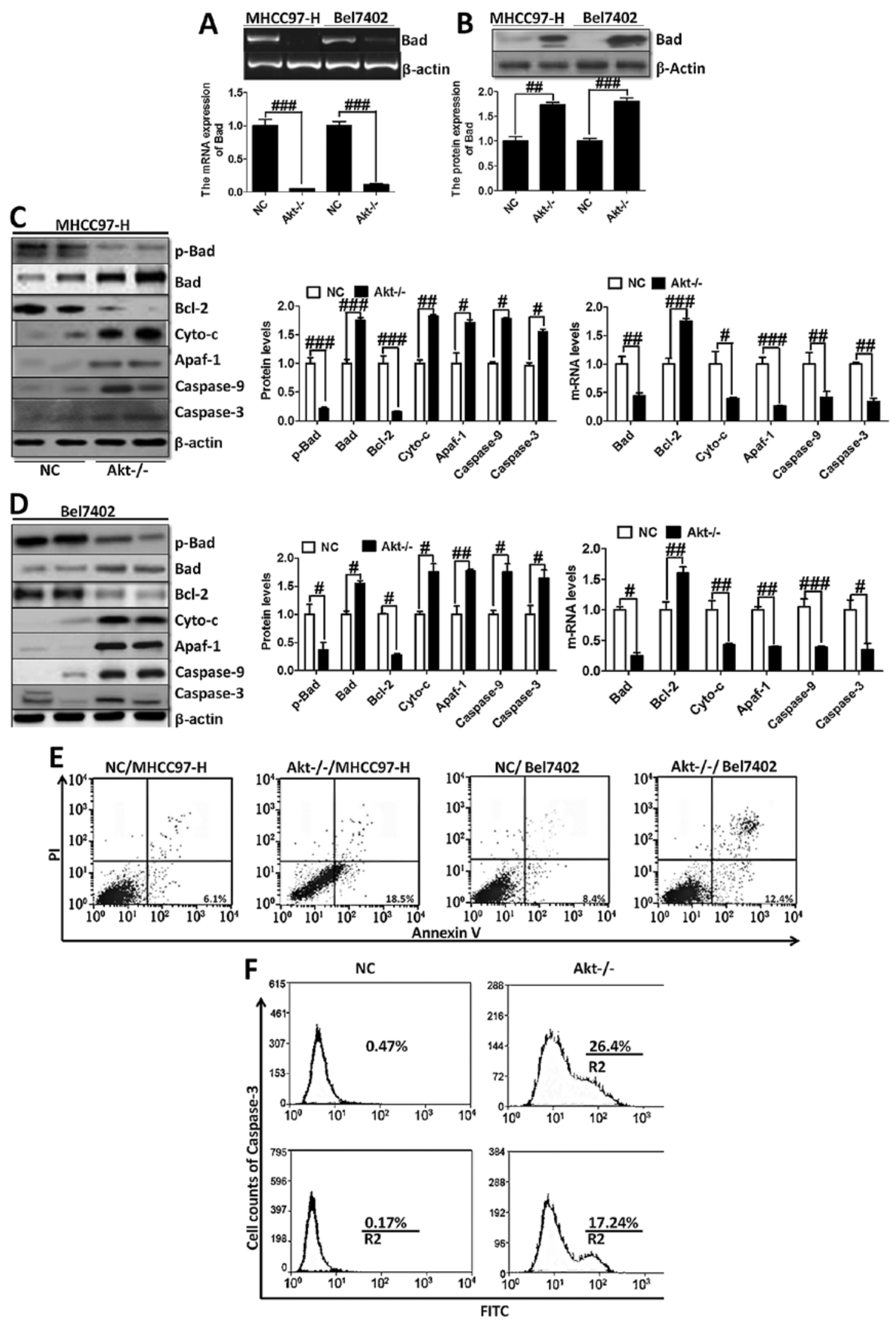

Figure 6. The effects of Akt on apoptosis via Bad and its downstream signals in liver cancer cell lines. (A) Bad mRNA levels; (B) Bad protein levels; (C) the expression of Bad signaling pathway via western blot analysis and RT-PCR analysis in MHCC97-H cells; (D) the expression of Bad signaling pathway via western blot analysis and RT-PCR analysis in MHCC97-H cells; (E) Akt-knockout induced significant apoptosis in liver cancer cell lines, determined by PI/Annexin V analysis; (F) caspase-3 activation assay with the CaspGLOW fluorescein active caspase-3 staining kit with FACS analysis. Data are expressed as the mean $\pm \mathrm{SEM} ;{ }^{\#} \mathrm{P}<0.05,{ }^{\# \#} \mathrm{P}<0.01,{ }^{\# \# \#} \mathrm{P}<0.001$ vs. $\mathrm{NC}$ group.

$\mathrm{NF}-\kappa \mathrm{B}$ in $\mathrm{Akt}^{-1-}$ liver cancer cells were lower than in the liver cancer cells, demonstrating that inhibition of overexpression of Akt could suppress the abnormal NF- $\mathrm{BB}$ expression, subsequently improving cytokine expression, including IL-1 $\beta$ and 


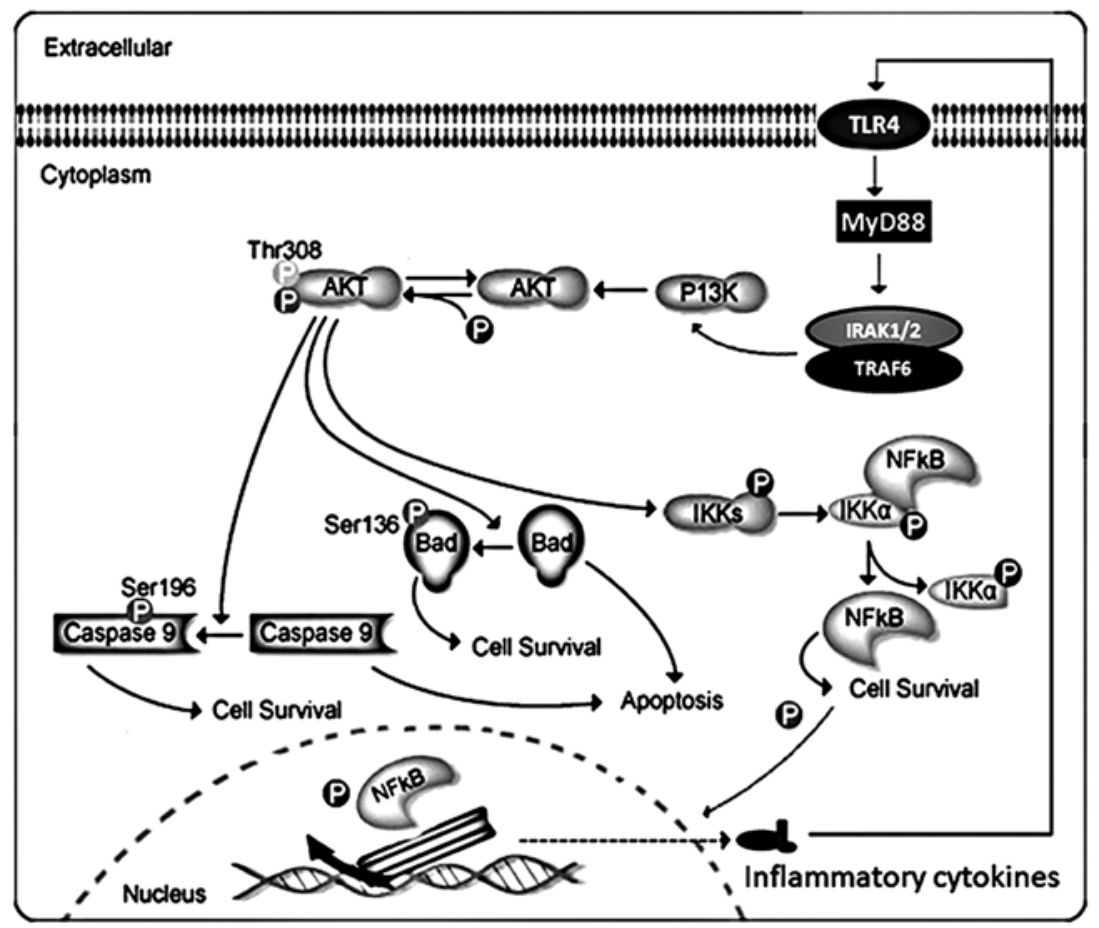

Figure 7. Proposed model. Knockout of Akt blocks the inflammatory signal and induces the apoptotic signaling pathway.

IL-18 (Fig. 3E and F). Furthermore, as shown in Fig. 4A, many cytokines were tested to further clarify the effect of Akt on the levels of inflammatory factors, which play an essential role in the progression of liver cancer. Then, overexpression of cytokines, in turn, further activated the TLR4/MyD88 signaling pathway, contributing to the downstream signals expression. Subsequently, activation of PI3K phosphorylated Akt, which led to the phosphorylation of NF- $\mathrm{BB}$, and finally intensified the inflammation response, which is a feature of cancer progression. A previous study (21) has also shown that expression of NF- $\mathrm{kB}$ results in cell survival. However, in the present study we supposed that knockout of Akt reduced $\mathrm{NF}-\kappa \mathrm{B}$ expression and lessened $\mathrm{p}-\mathrm{NF}-\mathrm{\kappa B}$ expression, accordingly damaging cancer cell survival and decreasing the feature of inflammation response in liver cancer cells, which was similar to the former research. Thus, the development and progression of liver cancer could be inhibited through knockout of Akt.

The $\mathrm{Bcl}-2$-associated death promoter (BAD) protein is a pro-apoptotic member of the Bcl-2 gene family which is involved in initiating apoptosis. BAD is a member of the BH3-only family, a subfamily of the Bcl-2 family (22). It does not contain a C-terminal transmembrane domain for outer mitochondrial membrane and nuclear envelope targeting, unlike most other members of the Bcl-2 family (23). When Bad is phosphorylated by Akt, it forms the Bad-(14-3-3) protein heterodimer. Bax are believed to initiate apoptosis by forming a pore in the mitochondrial outer membrane that allows cytochrome $c$ to escape into the cytoplasm and activate the pro-apoptotic caspase cascade (24). The anti-apoptotic Bcl-2 and Bcl-xL proteins inhibit cytochrome $c$ release through the mitochondrial pore and also inhibit activation of the cytoplasmic caspase cascade by cytochrome $c(25,26)$. These studies have suggested that activated Akt could phos- phorylate Bad, contributing to cell survival, and that caspase-9 and caspase-3, play essential roles in apoptosis. The results in the present study, similarly found that Akt-knockout liver cancer cells showed lower p-Bad expression and higher Bad expression, cutting down Bcl-xL expression and promoting the down stream signals, such as cytochrome $c$, Apaf-1, caspase-9 and caspase- 3 expression, subsequently contributing to apoptosis in cancer cells (Figs. 5 and 7). Western blot and RT-PCR analysis further confirmed the results that $\mathrm{Akt}^{-/-}$enhanced $\mathrm{Bad}$ expression. In addition, Annexin $\mathrm{V}$ and caspase-3 staining kit with FACS analysis (Fig. 6E and F) demonstrated that the Akt-knockout might affect caspase-3 in an indirect manner. As we mentioned above, the AKT signaling pathway might be associated with the progression of liver cancer. These data indicated that Akt is important upstream of NF- $\mathrm{kB}$ and $\mathrm{Bad}$ might impact inflammatory responses and apoptosis (Fig. 7). Thus, inhibition of Akt activation might be an essential strategy to suppress liver cancer progression via inflammation response and apoptosis.

Taken together, liver cancer has been emphasized as a threat to human health and life, particularly in developing countries. Considerable number of risk factors contribute to the occurrence and progression of liver cancer $(1-3,27)$. However, the underlying mechanisms of liver cancer are not yet clarified, and treatments for liver cancer are still lacking, thus, new strategies and studies are required for patients with the disease. In the present study, we investigated in a liver cancer animal model, and cells the Akt-regulated NF-kB and Bad signaling pathway and its downstream signals. Also, the Akt activation could be significantly upregulated in liver cancer cell lines, and knockout of Akt was able to reduce inflammation-related cytokine expression and apoptosisassociated signals, which might be potential indicators for clinical treatment. 


\section{Acknowledgements}

We are grateful for the support from the National Natural Science Foundation of China (81301923 and 81371902), and the National Natural Science Foundation of Fujian (2011D012 and 2015J01561). Medical Innovation Subject of Fujan Province of China (2012-CX-30).

\section{References}

1. Jemal A, Bray F, Center MM, Ferlay J, Ward E and Forman D: Global cancer statistics. CA Cancer J Clin 61: 69-90, 2011.

2. Kim YK, Lee GS, Jung EM, Hyun SH, Hwang WS and Jeung EB: Generation of fibroblasts overexpressing liver-specific PEPCK in a miniature pig model of human type 2 diabetes mellitus. Mol Med Rep 6: 45-50, 2012.

3. Rampone B, Schiavone B, Martino A, Viviano C and Confuorto G: Current management strategy of hepatocellular carcinoma. World J Gastroenterol 15: 3210-3216, 2009.

4. Xu G, Qi FZ, Zhang JH, Cheng GF, Cai Y and Miao Y: Metaanalysis of surgical resection and radiofrequency ablation for early hepatocellular carcinoma. World J Surg Oncol 10: 1632012, 2012.

5. Salhab M and Canelo R: An overview of evidence-based management of hepatocellular carcinoma: a meta-analysis. J Cancer Res Ther 7: 463-475, 2011.

6. Zhou XD: Recurrence and metastasis of hepatocellular carcinoma: progress and prospects. Hepatobiliary Pancreat Dis Int 1: 35-41, 2002.

7. Guan M, Zhou X, Soulitzis N, Spandidos DA and Popescu NC: Aberrant methylation and deacetylation of deleted in liver cancer-1 gene in prostate cancer: potential clinical applications. Clin Cancer Res 12: 1412-1419, 2006.

8. Lizarraga IM, Sugg SL, Weigel RJ and Scott-Conner CE: Review of risk factors for the development of contralateral breast cancer Am J Surg 206: 704-708, 2013.

9. Chapuis N, Tamburini J, Cornillet-Lefebvre P, Gillot L, Bardet V, Willems L, Park S, Green AS, Ifrah N, Dreyfus F, et al: Autocrine IGF-1/IGF-1R signaling is responsible for constitutive PI3K/ Akt activation in acute myeloid leukemia: therapeutic value of neutralizing anti-IGF-1R antibody. Haematologica 95: 415-423, 2010.

10. Muders MH, Zhang H, Wang E, Tindall DJ and Datta K: Vascular endothelial growth factor-C protects prostate cancer cells from oxidative stress by the activation of mammalian target of rapamycin complex-2 and AKT-1. Cancer Res 69: 6042-6048, 2009.

11. Opel D, Poremba C, Simon T, Debatin KM and Fulda S: Activation of Akt predicts poor outcome in neuroblastoma. Cancer Res 67: 735-745, 2007.

12. Chagpar RB, Links PH, Pastor MC, Furber LA, Hawrysh AD, Chamberlain MD and Anderson DH: Direct positive regulation of PTEN by the p85 subunit of phosphatidylinositol 3-kinase. Proc Natl Acad Sci USA 107: 5471-5476, 2010.
13. Lopez-Carballo G, Moreno L, Masia S, Perez P and Barettino D: Activation of the phosphatidylinositol 3-kinase/Akt signaling pathway by retinoic acid is required for neural differentiation of SH-SY5Y human neuroblastoma cells. J Biol Chem 277: 25297-25304, 2002.

14. Yang XW, Wang XL, Cao LQ, Jiang XF, Peng HP, Lin SM, Xue P and Chen D: Green tea polyphenol epigallocatechin-3-gallate enhances 5-fluorouracil-induced cell growth inhibition of hepatocellular carcinoma cells. Hepatol Res 42: 494-501, 2012.

15. Sugiyama M, Sakahara H, Torizuka T, Kanno T, Nakamura F, Futatsubashi $\mathrm{M}$ and Nakamura S: ${ }^{18}$ F-FDG PET in the detection of extrahepatic metastases from hepatocellular carcinoma. J Gastroenterol 39: 961-968, 2004.

16. Lee JH, Park JY, Kim do Y, Ahn SH, Han KH, Seo HJ, Lee JD and Choi HJ: Prognostic value of ${ }^{18}$ F-FDG PET for hepatocellular carcinoma patients treated with sorafenib. Liver Int 31: 1144-1149, 2011.

17. Paudyal B, Oriuchi N, Paudyal P, Tsushima Y, Higuchi T, Miyakubo M, Ishikita T, Nakajima T and Endo K: Clinicopathological presentation of varying ${ }^{18} \mathrm{~F}-\mathrm{FDG}$ uptake and expression of glucose transporter 1 and hexokinase II in cases of hepatocellular carcinoma and cholangiocellular carcinoma. Ann Nucl Med 22: 83-86, 2008

18. Ng KK, Poon RT, Lo CM, Yuen J, Tso WK and Fan ST: Analysis of recurrence pattern and its influence on survival outcome after radiofrequency ablation of hepatocellular carcinoma. J Gastrointest Surg 12: 183-191, 2008.

19. Berg $M$ and Soreide K: EGFR and downstream genetic alterations in KRAS/BRAF and PI3K/AKT pathways in colorectal cancer: implications for targeted therapy. Discov Med 14: 207-214, 2012.

20. Radisavljevic Z: AKT as locus of cancer angiogenic robustness and fragility. J Cell Physiol 228: 21-24, 2013.

21. DiDonato JA, Mercurio F, Karin M: NF- $\kappa$ B and the link between inflammation and cancer. Immunol Rev 246: 379-400, 2012.

22. Balogova L, Maslanakova M, Dzurova L, Miskovsky P and Stroffekova K: Bcl-2 proapoptotic proteins distribution in U-87 MG glioma cells before and after hypericin photodynamic action. Gen Physiol Biophys 32: 179-187, 2013.

23. Danial NN: BAD: undertaker by night, candyman by day. Oncogene 27 (Suppl 1): S53-S70, 2008.

24. Levine B, Sinha S and Kroemer G: Bcl-2 family members: dual regulators of apoptosis and autophagy. Autophagy 4: 600-606, 2008.

25. Stauffer SR: Small molecule inhibition of the Bcl-X(L)-BH3 protein-protein interaction: proof-of-concept of an in vivo chemopotentiator ABT-737. Curr Top Med Chem 7: 961-965, 2007.

26. Hojabrpour P, Waissbluth I, Ghaffari M, Cox ME and Duronio V: CaMKII-gamma mediates phosphorylation of BAD at Ser170 to regulate cytokine-dependent survival and proliferation. Biochem J 442: 139-149, 2012.

27. Yin DL, Jiang HC, Liang YJ, Meng XZ, Wang JB, Zheng TS and Liu LX: Precise hepatectomy guided by minimally invasive surgery: a novel strategy for liver resection. Hepatogastroenterology 59: 1951-1959, 2012. 